\title{
Refining the Klobuchar Ionospheric Model with Spherical Cap Harmonic Model for GNSS Positioning
}

\author{
K. Nishimoto, M. Ohashi, Y. Kubo and S. Sugimoto \\ Department of Electrical and Electronic Engineering, Ritsumeikan University \\ Noji-Higashi, Kusatsu City, Shiga 525-8577 Japan \\ Tel: +81-(0)77-561-5972, FAX: +81-(0)77-561-2663 \\ e-mail: ykubo@se.ritsumei.ac.jp
}

\begin{abstract}
Ionospheric delay is one of the major error sources in GNSS (Global Navigation Satellite System) positioning. For single frequency system, it is very important to effectively correct the ionospheric delay and so-called Klobuchar's ionospheric model has been widely used due to its well known properties such as computational simplicity. The Klobuchar model is one of the global ionospheric models, and it can correct about 50\% RMS ionospheric errors[1]. On the other hand, there are some models that can achieve better accuracy than the Klobuchar model, the SCHA (Spherical Cap Harmonics Analysis) model is one of such models. By the SCHA model, it has been reported that the ionospheric delays can be well modeled in the regional area such as the sky over Japan[2]. However, comparing with the Klobuchar model, the SCHA model needs more number of parameters and computational burden. Therefore, in this paper, a method to generate the parameters of the Klobuchar model from the SCHA model is proposed. By this method, the regional ionospheric model that is not only simple and easy operational like the Klobuchar model but also regionally accurate like the SCHA model is obtained.
\end{abstract}

\section{Introduction}

Klobuchar model is built on a constant offset term at night and a simple half-cosine function centered about 14:00 local time. The amplitude of the cosine term and its period, are function of latitude, ordered by geomagnetic, rather than geographic, latitude and are represented in the algorithm by third degree polynomials. The coefficients of these polynomials are transmitted as part of the satellite message [3]-[4]. The Klobuchar Ionospheric model has been widely used in the world. In contrast, SCHA model has been reported that the ionospheric delays can be well modeled in the sky over Japan. This paper proposes methods to refine the Klobuchar ionospheric coefficients using SCHA model. Namely, the Klobuchar ionospheric coefficients refined by the proposed methods have better accuracy than conventional coefficients in the sky over Japan[5].

\section{GNSS Regression Models}

By using a dual frequency receiver, typically four types of measurements are obtained, i.e. $\mathrm{C} / \mathrm{A}$ and $\mathrm{P}(\mathrm{Y})$ code pseudoranges $\rho_{C A, k}^{p}, \rho_{P Y, k}^{p}$ and L1 and L2 band carrier phases $\Phi_{L 1, k}^{p}, \Phi_{L 2, k}^{p}$ as follows[5]-[8]:

$$
\begin{gathered}
\rho_{C A, k}^{p}=r_{k}^{p}+c\left(\delta t_{k}-\delta t^{p}\right)+\delta I_{k}^{p}+\delta T_{k}^{p} \\
+\delta b_{C A, k}+\delta b_{C A}^{p}+e_{C A, k}^{p} \\
\rho_{P Y, k}^{p}=r_{k}^{p}+c\left(\delta t_{k}-\delta t^{p}\right)+\frac{f_{1}^{2}}{f_{2}^{2}} \delta I_{k}^{p}+\delta T_{k}^{p} \\
+\delta b_{P Y, k}+\delta b_{P Y}^{p}+e_{P Y, k}^{p} \\
\Phi_{L 1, k}^{p}=r_{k}^{p}+c\left(\delta t_{k}-\delta t^{p}\right)-\delta I_{k}^{p}+\delta T_{k}^{p} \\
+\lambda_{1} N_{L 1, k}^{p}+\delta b_{L 1, k}+\delta b_{L 1}^{p}+\varepsilon_{L 1, k}^{p} \\
\Phi_{L 2, k}^{p}=r_{k}^{p}+c\left(\delta t_{k}-\delta t^{p}\right)-\frac{f_{1}^{2}}{f_{2}^{2}} \delta I_{k}^{p}+\delta T_{k}^{p} \\
+\lambda_{2} N_{L 2, k}^{p}+\delta b_{L 2, k}+\delta b_{L 2}^{p}+\varepsilon_{L 2, k}^{p}
\end{gathered}
$$

where $c$ is speed of light, $f_{*}$ and $\lambda_{*}$ are the frequency and wave length of $\mathrm{L}_{*}$ band carrier signal respectively. $\quad\left\{\delta b_{C A, k}, \delta b_{P Y, k}, \delta b_{L 1, k}, \delta b_{L 2, k}\right\}$ and $\left\{\delta b_{C A}^{p}, \delta b_{P Y}^{p}, \delta b_{L 1}^{p}, \delta b_{L 2}^{p}\right\}$ are receiver's and satellite's hardware biases. $r_{k}^{p}$ is the geometric distance between the receiver $k$ and the satellite $p . \delta t_{k}$ and $\delta t^{p}$ are the clock errors of the receiver $k$ and the satellite $p . \delta I_{k}^{p}$ and $\delta T_{k}^{p}$ are ionospheric and troposphere delays. $N_{L 1, k}^{p}$ and $N_{L 2, k}^{p}$ are phase ambiguities. $e$ and $\varepsilon$ are measurement errors. Also, $r_{k}^{p}$ is the geometric distance between the receiver $k$ and satellite $p\left(p=1, \cdots, n_{s}\right)$. Namely,

$$
r_{k}^{p}=\sqrt{\left(x_{k}-x^{p}\right)^{2}+\left(y_{k}-y^{p}\right)^{2}+\left(z_{k}-z^{p}\right)}
$$

where $k \equiv\left[x_{k}, y_{k}, z_{k}\right]^{T}$ and $s^{p} \equiv\left[x^{p}, y^{p}, z^{p}\right]^{T}$ are the known receiver position and the $p$-th satellite position, respectively. $n_{s}$ is the number of visible satellites.

\subsection{Vector Representation}

(1)-(4) can be expressed by the following vector regression equation for $n_{s}$ satellites.

$$
\boldsymbol{y}_{k}=H_{k} \boldsymbol{\theta}_{k}+\boldsymbol{v}_{k}
$$


where

$$
\begin{aligned}
& \boldsymbol{y}_{k} \equiv\left[\begin{array}{cccc}
\rho_{C A, k}^{T} & \rho_{P Y, k}^{T} & \Phi_{L 1, k}^{T} & \Phi_{L 2, k}^{T}
\end{array}\right]^{T} \\
& H_{k}=\left[\begin{array}{ccccccc}
I & \mathbf{1} & -I & \mathbf{1} & & & \\
I & \mathbf{1} & -I & & \mathbf{1} & & \\
I & \mathbf{1} & -I & & & \mathbf{1} & \\
I & \mathbf{1} & -I & & & & \mathbf{1}
\end{array}\right.
\end{aligned}
$$

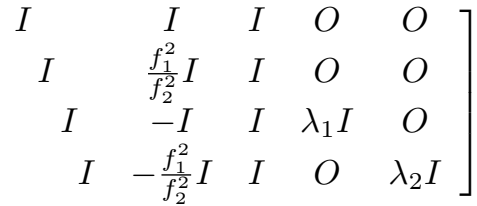

$$
\begin{aligned}
& \boldsymbol{\theta}_{k}=\left[\begin{array}{lllll}
\boldsymbol{r}_{k}^{T} & \boldsymbol{c} \boldsymbol{\delta} \boldsymbol{t}_{k}^{T} & \boldsymbol{c} \boldsymbol{\delta} \boldsymbol{t}^{s T} & \boldsymbol{\delta} \boldsymbol{b}_{k}{ }^{T} & \boldsymbol{\delta} \boldsymbol{b}^{s T}
\end{array}\right. \\
& \left.\begin{array}{llll}
\boldsymbol{\delta}_{k}{ }^{T} & \boldsymbol{\delta} \boldsymbol{T}_{k}{ }^{T} & \boldsymbol{\delta} \boldsymbol{N}_{L 1, k} & \boldsymbol{\delta} \boldsymbol{N}_{L 2, k}{ }^{T}
\end{array}\right]^{T} \\
& \boldsymbol{v}_{k} \equiv\left[\begin{array}{llll}
\boldsymbol{e}_{C A, k}^{T} & \boldsymbol{e}_{P Y, k}^{T} & \boldsymbol{\varepsilon}_{L 1, k}^{T} & \boldsymbol{\varepsilon}_{L 2, k}^{T}
\end{array}\right]^{T}
\end{aligned}
$$

and $\mathbf{1} \equiv[1, \cdots, 1], n_{s} \times 1$ vector, $I$ denote the $n_{s} \times n_{s}$ unit matrix.

\subsection{Linear Transform}

By applying the following linear transformation, several unknown parameters can be canceled.

$$
D \boldsymbol{y}_{k}=D H_{k} \boldsymbol{\theta}_{k}+D \boldsymbol{v}_{k}
$$

where,

$$
D \equiv\left[\begin{array}{cccc}
-I & I & O & O \\
O & O & -I & I
\end{array}\right] .
$$

The components of the left hand side of Eq.(11) can be calculated as

$$
\begin{aligned}
\rho_{P Y, k}^{p}-\rho_{C A, k}^{p}= & \delta b_{P Y, k}-\delta b_{C A, k}+\delta b_{P Y}^{p}-\delta b_{C A}^{p} \\
& +\left(\frac{f_{1}^{2}}{f_{2}^{2}}-1\right) \times \delta I \\
& +e_{P Y, k}^{p}-e_{C A, k}^{p} \\
\Phi_{L 2, k}^{p}-\Phi_{L 1, k}^{p}= & \delta b_{L 1, k}-\delta b_{L 2, k}+\delta b_{L 1}^{p}-\delta b_{L 1}^{p} \\
& -\left(\frac{f_{1}^{2}}{f_{2}^{2}}-1\right) \times \delta I \\
& +\lambda_{2} N_{L 2, k}-\lambda_{1} N_{L 1, k} \\
& +\varepsilon_{L 2, k}^{p}-\varepsilon_{L 1, k}^{p} .
\end{aligned}
$$

From Eqs.(13) and (14), the following vector regression equation for $n_{s}$ satellites can be obtained.

$$
\begin{aligned}
& {\left[\begin{array}{c}
\boldsymbol{\rho}_{P Y, k}-\boldsymbol{\rho}_{C A, k} \\
\boldsymbol{\Phi}_{L 2, k}-\boldsymbol{\Phi}_{L 1, k}
\end{array}\right] } \\
= & {\left[\begin{array}{ccc}
\left(\frac{f_{1}^{2}}{f_{2}^{2}}-1\right) I & I & O \\
-\left(\frac{f_{1}^{2}}{f_{2}^{2}}-1\right) I & O & I
\end{array}\right]\left[\begin{array}{c}
\boldsymbol{\delta} I_{k} \\
\boldsymbol{B}_{P Y-C A, k} \\
\boldsymbol{B}_{L 2-L 1, k}
\end{array}\right] } \\
& +\left[\begin{array}{c}
\boldsymbol{e}_{P Y, k}+\boldsymbol{e}_{C A, k} \\
\boldsymbol{\varepsilon}_{L 2, k}+\boldsymbol{\varepsilon}_{L 1, k}
\end{array}\right]
\end{aligned}
$$

where,

$$
\begin{aligned}
\boldsymbol{B}_{P Y-C A, k}=\mathbf{1}( & \left.\boldsymbol{\delta} \boldsymbol{b}_{P Y, k}-\boldsymbol{\delta} \boldsymbol{b}_{C A, k}\right) \\
& +\boldsymbol{\delta} \boldsymbol{b}_{P Y}^{s}-\boldsymbol{\delta} \boldsymbol{b}_{C A}^{s} \\
\boldsymbol{B}_{L 2-L 1, k}=\mathbf{1}( & \left.\boldsymbol{\delta} \boldsymbol{b}_{L 2, k}-\boldsymbol{\delta} \boldsymbol{b}_{L 1, k}\right)+\boldsymbol{\delta} \boldsymbol{b}_{L 2}^{s} \\
& -\boldsymbol{\delta} \boldsymbol{b}_{L 1}^{s}+\lambda_{2} \boldsymbol{N}_{L 2}^{s}-\lambda_{1} \boldsymbol{\delta} \boldsymbol{b}_{L 1}^{s} .
\end{aligned}
$$

Eq. (15) is the fundamental observation equation of the ionospheric delay. By obtaining the ionospheric delay of Eq. (15) from multiple satellites, receivers and epochs, the ionospheric delays are modeled.

\section{$3 \quad$ Ionospheric Models}

There exist several ionospheric models for correcting the ionospheric delays in GNSS position calculation. The Klobuchar model is the most popular for civil, stand alone, i.e. the point positioning, users because the model parameters of the Klobuchar model are broadcast from GPS satellites. It is reported that about 50 [\%] RMS ionospheric delays can be corrected by using the Klobuchar model.

On the other hand, the authors have proposed the Spherical Cap Harmonic Analysis (SCHA) model as more precise model than the Klobuchar model. In this paper, we consider the method to refine the Klobuchar model parameter by using the SCHA model.

In this section, the Klobuchar and SCHA models are briefly reviewed.

\subsection{Klobuchar Ionospheric Model}

In the Klobuchar model, it is assumed that all free electrons of the ionosphere are in a shell located at a fixed altitude of $350 \mathrm{~km}$ above Earth's surface, and this model is built on a constant offset term at night and simple half period cosine function that centered about 14:00 local time during the day [3]. Fig.1 illustrates this model.

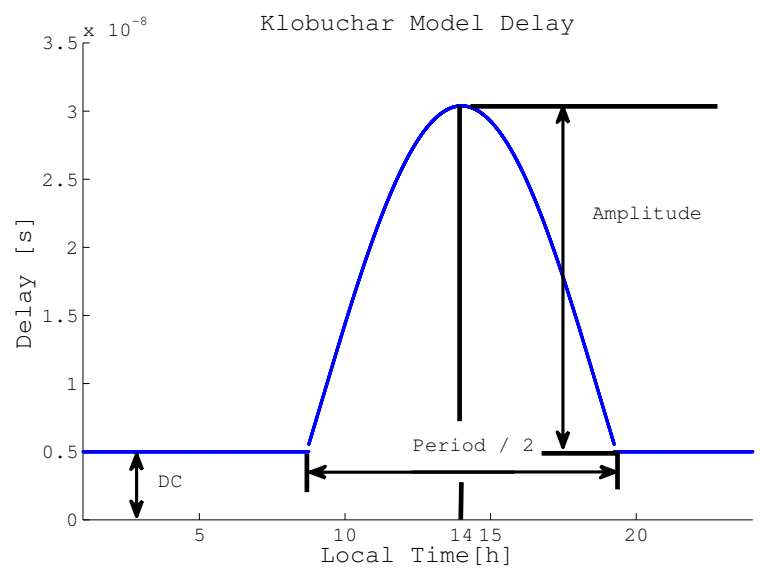

Fig. 1: Example of Ionosphere delay calculated by Klobuchar model 
According to [3], the ionospheric delay $T_{i o n o, k l}$ of the Klobuchar model can be calculated by the following algorithm.

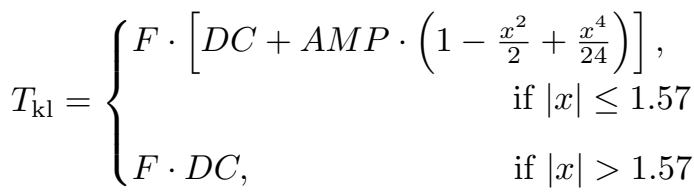

$$
\begin{aligned}
& x=\frac{2 \pi(t-50400)}{P E R} \\
& A M P=\left\{\begin{array}{l}
\sum_{n=0}^{3} \alpha_{n} \phi_{m}^{n} \\
\text { if } A M P<0, \quad A M P=0
\end{array}\right. \\
& \text { PER }=\left\{\begin{array}{l}
\sum_{n=0}^{3} \beta_{n} \phi_{m}^{n} \\
\text { if } P E R<72000, \quad P E R=72000
\end{array}\right. \\
& F=\operatorname{cosec}\left[\arcsin \left(\frac{R_{E}}{R_{E}+H} \sin (z)\right)\right] \\
& \phi_{m}=\phi_{i}+0.064 \cos \left(\lambda_{i}-1.617\right) \\
& \lambda_{i}=\lambda_{u}+\frac{\Psi \sin A}{\cos \phi_{i}} \\
& \phi_{i}=\left\{\begin{array}{l}
\phi_{u}+\Psi \cos A \\
\quad \text { if }\left|\phi_{i}\right|>0.416, \quad \phi_{i}=0.416 \phi_{i} /\left|\phi_{i}\right|
\end{array}\right. \\
& \Psi=\frac{0.0137}{E+0.11}-0.022 \\
& t= \begin{cases}4.32 \times 10^{4} \lambda_{i}+t_{G P S} \\
\text { if } t>86400, \quad t=t-86400 \\
\text { if } t<0, & t=t+86400\end{cases}
\end{aligned}
$$

where, $D C$ is the night time constant offset term of the vertical time-delay $\left(5 \times 10^{-9} s\right)$. $A M P$ is the amplitude term of the cosine function. $P E R$ is the period of the cosine function. $F$ is the obliquity factor projecting vertical ionospheric delay into the line of sight. $\phi_{m}$ is the geomagnetic latitude of the earth projection of the ionospheric pierce point. $x$ is the radian of the cosine function. $\phi_{i}$ is the geodetic latitude of the earth projection of the ionospheric pierce point. $\lambda_{i}$ is the geodetic longitude of the earth projection of the ionospheric pierce point. $A$ is the azimuth angle of the satellite. $\phi_{u}$ is the user geodetic latitude in WGS-84. $\lambda_{u}$ is the user geodetic longitude in WGS- 84 . $\Psi$ is Earth's central angle between the user position and and the earth projection of the ionospheric pierce point. $E$ is elevation angle of the satellite. $R_{E}$ is Earth radius. $H$ is ionospheric height. $t$ is local time. $t_{G P S}$ is GPS satellite clock time. The parameters $\alpha_{n}, \beta_{n}(n=0,1,2,3)$ are broadcast from GPS satellites and updated once each ten days, or sometimes more frequently.

\subsection{Spherical Cap Harmonics Analysis Model of Ionospheric Delay}

The ionospheric SCHA model can be written as[2]

$$
\begin{gathered}
\delta I=\frac{40.3 \times 10^{16}}{f^{2}} \times\left[F \times \sum_{n=0}^{n_{\max }} \sum_{m=0}^{n}\left\{a_{n m} \cos m s_{I P}\right.\right. \\
\left.\left.+b_{n m} \sin m s_{I P}\right\} P_{n}^{m}\left(\sin \beta_{I P}\right)\right]
\end{gathered}
$$

where, $f$ is the frequency of $\mathrm{L}_{1}$ band carrier signal. $P_{n}^{m}$ is the associated Legendre function in SCHA. $\beta_{I P}$ and $\alpha_{I P}$ denote the latitude and the longitude of the ionospheric pierce point in the sun-fixed coordinate system. Namely, $s_{I P}=\lambda_{I P}-\lambda_{0}$, where $\lambda_{0}$ is the longitude of the Sun. $a_{n m}$ and $b_{n m}$ are the parameters of SCHA. These parameters can be determined by the least squares method with the ionospheric measurement obtained from Eqs. (1)-(4). Once the parameters are determined, the ionospheric time delay at the desired point can be obtained by

$$
\begin{gathered}
T_{\mathrm{sp}}=\frac{40.3 \times 10^{16}}{c \cdot f^{2}} \times\left[F \times \sum_{n=0}^{n_{\max }} \sum_{m=0}^{n}\left\{a_{n m} \cos m s_{I P}\right.\right. \\
\left.\left.+b_{n m} \sin m s_{I P}\right\} P_{n}^{m}\left(\sin \beta_{I P}\right)\right] .
\end{gathered}
$$

\section{Refining Klobuchar Model}

As mentioned above, the Klobuchar model is widely used due to its worldwide coverage and simple implementation aspects. However its performance is moderate such that about only $50 \%$ ionospheric effect can be removed. On the other hand, by using the SCHA method, it is reported that the regional ionosphere such as the sky over Japan can be modeled more precisely. Therefore, the idea of this paper is to refine the parameters of the Klobuchar model by using the SCHA model, and construct the ionospheric model that is not only simple but also precise in the regional area. For this purpose, 9 parameters of the Klobuchar model, i.e. $D C, \alpha_{n}, \beta_{n}(n=0, \ldots, 3)$ in Eqs. (18), (20) and (21) are estimated by using ionospheric delay information obtained from the SCHA model.

\subsection{Refining $D C$ Parameter}

From Eq. (18), we can see that the vertical ionospheric delay is modeled by the constant value $D C=$ $5.0 \times 10^{-9}$ when $|x|>1.57$. The value of $x$ depends on the local time $t$ and $|x|>1.57$ typically in the night time (between about 21:00 and 5:00). And in the original Klobuchar model specification, $D C$ value is always fixed at $5.0 \times 10^{-9}$. For example, the black line with symbol $\nabla$ in Fig. 2 shows the vertical ionospheric delay at the point of $(35.74 \mathrm{~N}, 137.94 \mathrm{E})$ obtained from the Klobuchar model, and we can see that the ionospheric delays from 12:00 to 18:00 (UTC) are the same. 


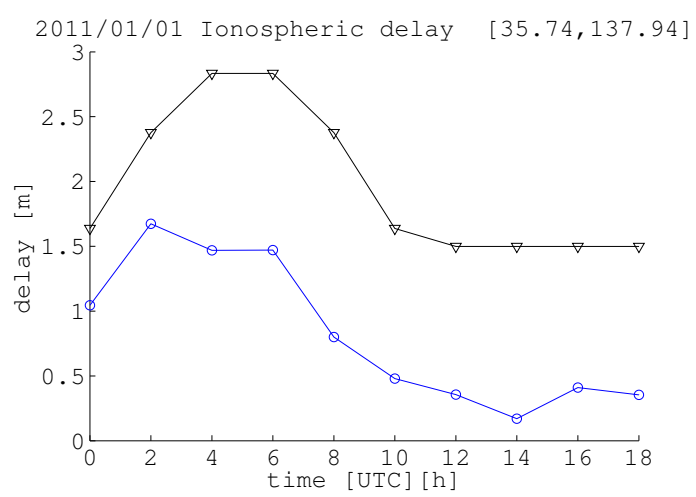

Fig. 2: Ionospheric delay obtained from Klobuchar model $(\nabla)$ and SCHA model $(\bigcirc)$ on Jan. 1, 2011 at $(35.74 \mathrm{~N}, 137.94 \mathrm{E})$

On the other hand, the blue line with symbol $\bigcirc$ in Fig. 2 shows the ionospheric delay obtained from the SCHA model, and we can see that the ionospheric delay in the night time is much smaller than Klobuchar fixed value. Consequently, the ionospheric delay of the Klobuchar model is quite bigger through the day.

Therefore the $D C$ parameter should be refined to improve the model accuracy. In this paper, the $D C$ parameter is refined by taking the average of the night time ionospheric delays obtained from the SCHA model. Now assume that the ionospheric delays of the SCHA model are calculated at certain number of points within the area focused on at a certain time interval. Let $N_{\text {data }}$ be the total number of ionospheric delays obtained from the SCHA model in the night time. Then the refined $D C$ parameter can be formulated as:

$$
D C_{\text {new }}=\frac{1}{N_{\text {data }}} \sum_{i=1}^{N_{\text {data }}} T_{\text {night }}^{i},
$$

where $D C_{\text {new }}$ is the refined $D C$ parameter, $T_{\text {night }}^{i}$ is the

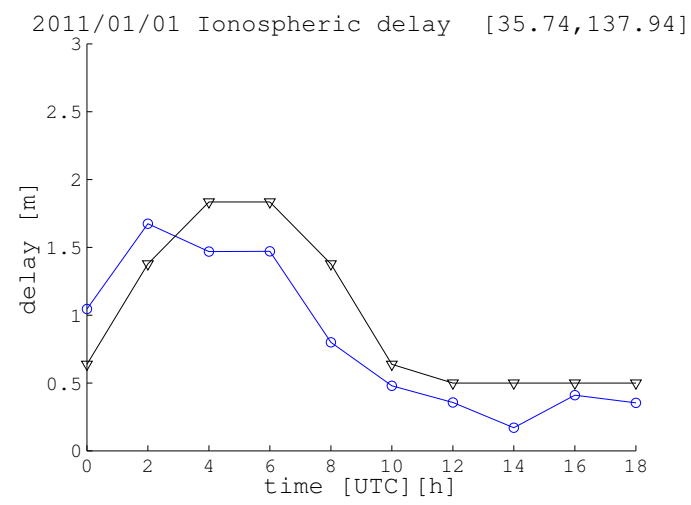

Fig. 3: Ionospheric delay obtained from Klobuchar model with refined $D C$ parameter $(\nabla)$ and SCHA model $(\bigcirc)$ on Jan. 1, 2011 at $(35.74 \mathrm{~N}, 137.94 \mathrm{E})$ $i$-th ionospheric delay obtained from the SCHA model in the night time.

For example, by using the Klobuchar and SCHA model on January 1, 2011, i.e. the same data of Fig. 2, the refined $D C$ parameter is obtained by Eq. (30). The ionospheric delay at the point $(35.74 \mathrm{~N}, 137.94 \mathrm{E})$ obtained by the Klobuchar model with refined $D C$ parameter is plotted by the black line with $\nabla$ symbol in Fig. 3. From Fig. 3, we can see that the Klobuchar model is quite improved by using the refined $D C$ parameter.

\subsection{Refining Parameters $\alpha$ and $\beta$}

Next, the estimation method of the parameters $\alpha_{n}$, $\beta_{n}, n=1, \ldots, 3$ is considered. For this purpose, Eq. (18) is expanded by the Taylor series expansion [9] around the approximations $\tilde{\alpha}_{n}$ and $\tilde{\beta}_{n}$, and the second and higher order terms are neglected. Then the linearized equation of Eq. (18) can be written as

$$
T_{\mathrm{kl}} \approx \tilde{T}_{\mathrm{kl}}+\sum_{n=0}^{3} \frac{\partial T_{\mathrm{kl}}}{\partial \alpha_{n}} d \alpha_{n}+\sum_{n=0}^{3} \frac{\partial T_{\mathrm{kl}}}{\partial \beta_{n}} d \beta_{n},
$$

where $d \alpha_{n} \equiv \alpha_{n}-\tilde{\alpha}_{n}, d \beta_{n} \equiv \beta_{n}-\tilde{\beta}_{n}$ and $T_{\mathrm{kl}}$ can be calculated from Eqs. (18)-(21) with $\tilde{\alpha}_{n}$ and $\tilde{\beta}_{n}$.

$$
\begin{aligned}
\frac{\partial T_{\mathrm{kl}}}{\partial \alpha_{n}}=F & \cdot\left(1-\frac{x^{2}}{2}+\frac{x^{4}}{2}\right) \cdot \phi_{m}^{n} \\
\frac{\partial T_{\mathrm{kl}}}{\partial \beta_{n}}=F & \cdot A M P \cdot\left[\frac{4 \pi^{2} \cdot(t-5400)^{2}}{P E R^{3}}\right. \\
& \left.-\frac{8}{3} \cdot \frac{\pi^{4}(t-50400)^{4}}{P E R^{5}}\right] \cdot \phi_{m}^{n}
\end{aligned}
$$

When samples of ionospheric delays at $K$ points are taken from the SCHA model, totally $K$ measurement for Eq. (31) are obtained. They can be expressed in the vector-matrix form as follows:

$$
\left[\begin{array}{ll}
A & B
\end{array}\right] \cdot\left[\begin{array}{l}
\boldsymbol{d} \boldsymbol{\alpha} \\
\boldsymbol{d} \boldsymbol{\beta}
\end{array}\right]=d
$$

where,

$$
\begin{aligned}
& A=\left[\begin{array}{ccc}
\frac{\partial T_{\mathrm{kl}}^{1}}{\partial \alpha_{0}} & \cdots & \frac{\partial T_{\mathrm{kl}}^{1}}{\partial \alpha_{3}} \\
\frac{\partial T_{\mathrm{kl}}^{2}}{\partial \alpha_{0}} & \cdots & \frac{\partial T_{\mathrm{kl}}^{2}}{\partial \alpha_{3}} \\
\vdots & & \vdots \\
\frac{\partial T_{\mathrm{kl}}^{K}}{\partial \alpha_{0}} & \cdots & \frac{\partial T_{\mathrm{kl}}^{K}}{\partial \alpha_{3}}
\end{array}\right] \\
& B=\left[\begin{array}{ccc}
\frac{\partial T_{\mathrm{kl}}^{1}}{\partial \beta_{0}} & \cdots & \frac{\partial T_{\mathrm{kl}}^{1}}{\partial \beta_{3}} \\
\frac{\partial T_{\mathrm{kl}}^{2}}{\partial \beta_{0}} & \cdots & \frac{\partial T_{\mathrm{kl}}^{2}}{\partial \beta_{3}} \\
\vdots & & \vdots \\
\frac{\partial T_{\mathrm{kl}}^{K}}{\partial \beta_{0}} & \cdots & \frac{\partial T_{\mathrm{kl}}^{K}}{\partial \beta_{3}}
\end{array}\right] \\
& \boldsymbol{d} \boldsymbol{\beta}=\left[\begin{array}{llll}
d \alpha_{0} & d \alpha_{1} & d \alpha_{2} & d \alpha_{3}
\end{array}\right]^{T} \\
& \boldsymbol{d} \boldsymbol{\alpha}=\left[\begin{array}{llll}
d \beta_{0} & d \beta_{1} & d \beta_{2} & d \beta_{3}
\end{array}\right]^{T} \\
& \boldsymbol{d}=\left[\begin{array}{lll}
d_{1} & \cdots & d_{K}
\end{array}\right]^{T}
\end{aligned}
$$


where a superscript which indicates the sampling point of the SCHA model is added to the variable $T_{\mathrm{kl}}$, and $d_{k} \equiv T_{\mathrm{sp}}^{k}-\tilde{T}_{\mathrm{kl}}^{k}(k=1,2, \cdots, K)$. Then by applying the least squares method to Eq. (34), the estimates of $d \alpha_{0}, \ldots, d \alpha_{3}, d \beta_{0}, \ldots, d \beta_{3}$ are obtained. These refined parameters $\alpha_{n}=\tilde{\alpha}_{n}+d \alpha_{n}$ and $\beta_{n}=\tilde{\beta}_{n}+d \beta_{n}$ can be used similarly to the Klobuchar parameter. Finally, the refined and ordinary Klobuchar models are compared in the GPS positioning by using real receiver data.

\section{Experiments and Results}

\subsection{Estimation SCHA model}

We estimated SCHA model parameters on June 1st, 2011, by using GEONET data for 2 hours and the sampling time interval of data was 10 minutes. The total number of the used GEONET stations was 42. Fig.(4) shows the locations of the used stations.

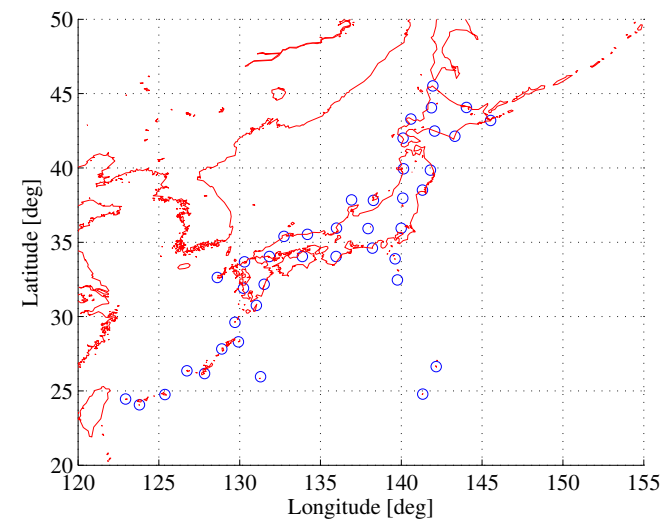

Fig. 4: GEONET stations for SCHA model parameter estimation

\subsection{Experimental Results}

In order to evaluate the effectiveness of the refined Klobuchar coefficients, the single point positioning accuracies applied by the following three types of ionospheric correction information were compared.

- Method 1: Klobuchar model with originally broadcast parameters

- Method 2: Klobuchar model with parameters refined by proposed method

- Method 3: Klobuchar model with parameters refined by using the SCHA model of the previous day (May 31, 2011)

The original Klobuchar model parameters are typically updated every ten days. In other words, the original Klobuchar model remains effective for ten days. Furthermore, in the actual operation, only the past SCHA model can be applied to refine the Klobuchar model. Therefore, in the Method 3 of this experiment,
Table 1: Original and refined Klobuchar model parameters

\begin{tabular}{|c|r|r|}
\hline Parameters & \multicolumn{1}{|c|}{ Refined } & \multicolumn{1}{c|}{ Original } \\
\hline \hline$\alpha_{0}$ & $2.9884 \mathrm{e}-8$ & $1.3040 \mathrm{e}-8$ \\
\hline$\alpha_{1}$ & $-2.4739 \mathrm{e}-8$ & $2.2350 \mathrm{e}-8$ \\
\hline$\alpha_{2}$ & $7.5105 \mathrm{e}-7$ & $-5.9600 \mathrm{e}-8$ \\
\hline$\alpha_{3}$ & $-1.1425 \mathrm{e}-6$ & $-1.1920 \mathrm{e}-7$ \\
\hline$\beta_{0}$ & $-1.5484 \mathrm{e}+5$ & $1.0440 \mathrm{e}+5$ \\
\hline$\beta_{1}$ & $8.3992 \mathrm{e}+6$ & $1.4750 \mathrm{e}+5$ \\
\hline$\beta_{2}$ & $-8.2882 \mathrm{e}+7$ & $-6.5540 \mathrm{e}+4$ \\
\hline$\beta_{3}$ & $2.6207 \mathrm{e}+8$ & $-4.5880 \mathrm{e}+5$ \\
\hline$D C$ & $4.6484 \mathrm{e}-9$ & $5.0000 \mathrm{e}-9$ \\
\hline
\end{tabular}

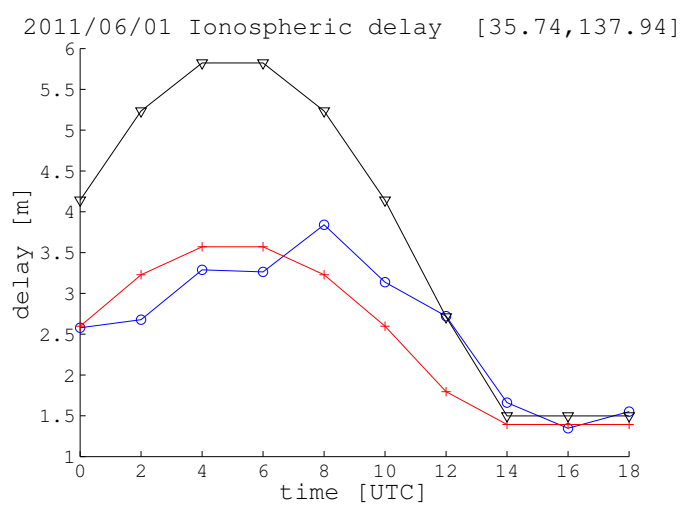

Fig. 5: Estimated ionospheric delay at Minowa town (35.740952N, 137.94386E) on June 1, 2011; $\nabla$ with black line: original Klobuchar model, + with red line: refined Klobuchar model, $\bigcirc$ with blue line: SCHA Model

the performance of the Klobuchar model with the parameters refined by the previous day's SCHA model are also investigated.

\section{Results on June 1, 2011}

By using the SCHA model on June 1, 2011, the refined Klobuchar model parameters were obtained. Table 1 shows the refined as well as the original parameters.

Fig. 5 shows the ionospheric delays that were calculated by refined parameters, original parameters and SCHA model on June 1, 2011. Fig. 6 shows the ionospheric delay that were calculated by refined coefficients. From Fig. 6, it can be seen that the ionospheric delay increases from high to low latitude due to solar activity.

Next, the positioning accuracies were evaluated. The observation data were obtained from GEONET reference station (Kushimoto, Seikai, and Wakkanai) on June 1, 2011, from 9:00:00 to 17:59:59 (Local Time). At the stations, the Trimble NETRS receivers with TRM29659.00 antennas observed the GPS signals at 
ospheric delay on 135 degree east longitude

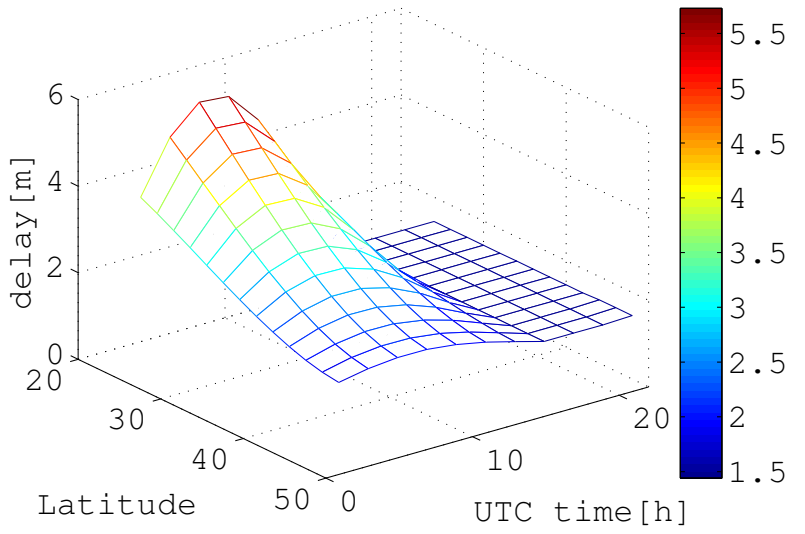

Fig. 6: Delay at 135 degree east longitude on June 1, 2011.

every 30 seconds. In the experiments, an elevation cutoff angle of 10 [deg] was applied[10]. The observation of the satellite positions and satellite clocks errors were obtained from the broadcast ephemeris. The positioning errors of the station "Kushiomoto" are shown in Figs. 7-9. Fig. 7 shows the position errors with original parameters. Fig. 8 shows the position errors with refined parameters. Fig. 9 shows the position errors with previous day's refined parameters.

The statics of errors in positioning at three stations are summarized in Tables 2-4. In the tables, the bias, standard deviation and RMS values of positioning errors are shown for horizontal plane and up directions. From Tables 2-4, we can see that the positioning results applied by the ionospheric delay correction based on the refined Klobuchar model (Method 2) are improved comparing with the results based on the original Klobuchar model (Method 1). We can also see that the position-

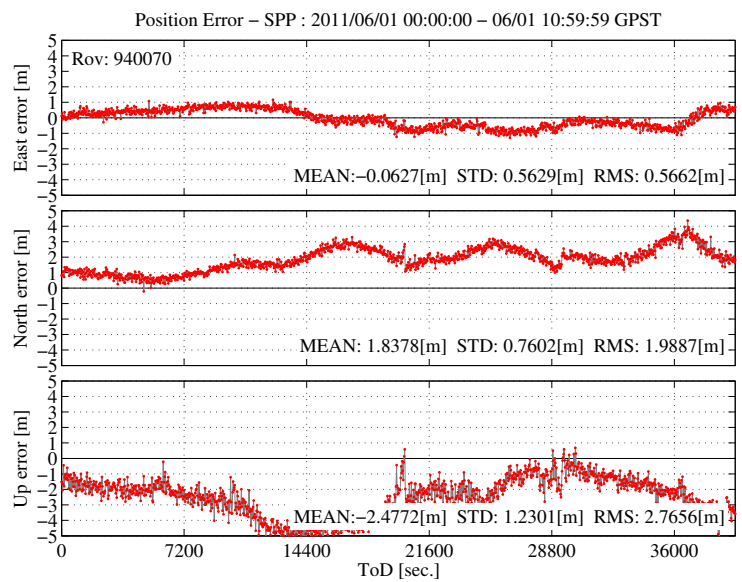

Fig. 7: Positioning errors (Method 1) at Kushimoto GEONET station

ing accuracy based on the Klobuchar model refined by the SCHA model of the previous day (Method 3) is slightly degraded than Method2. However the results of Method 3 show better performance than the original Klobuchar model (Method 1).

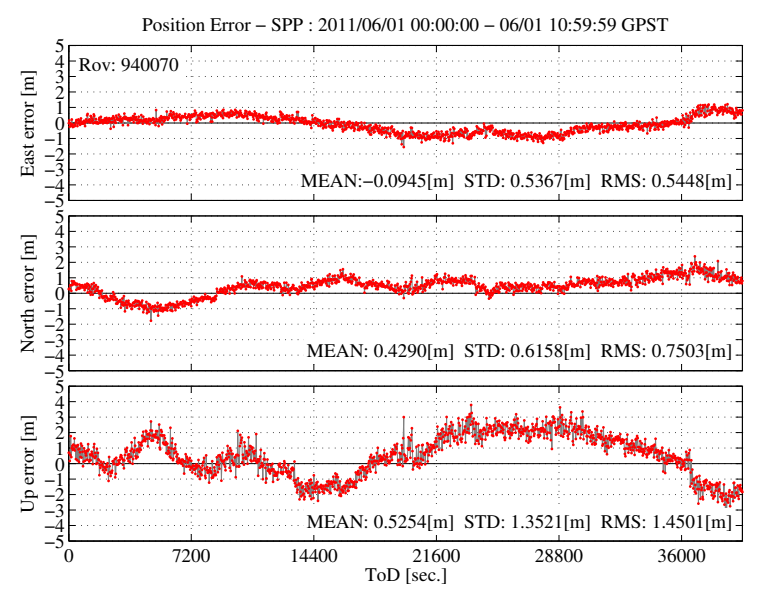

Fig. 8: Positioning errors (Method 2) at Kushimoto GEONET station

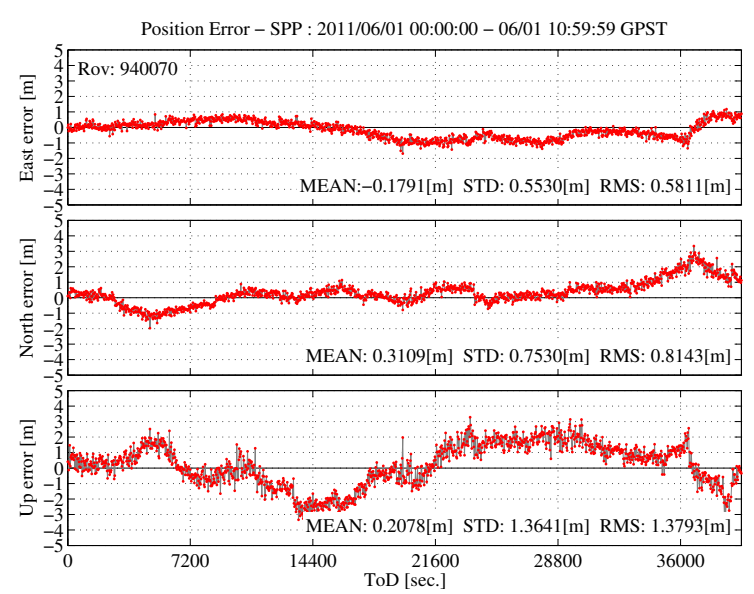

Fig. 9: Positioning errors (Method 3) at Kushimoto GEONET station

Table 2: Result Table Kushimoto

\begin{tabular}{|c|c|c|c|}
\hline Kushimoto & Method 1 & Method2 & Method 3 \\
\hline \hline 2D MEAN[m] & 1.8388 & 0.4393 & 0.3588 \\
\hline 2D STD $[\mathrm{m}]$ & 0.9460 & 0.8169 & 0.9342 \\
\hline 2D RMS[m] & 2.0677 & 0.9272 & 1.0004 \\
\hline UP MEAN[m] & -2.4772 & 0.5254 & 0.2078 \\
\hline UP STD $[\mathrm{m}]$ & 1.2301 & 1.3521 & 1.3641 \\
\hline UP RMS[m] & 2.7656 & 1.4501 & 1.3793 \\
\hline
\end{tabular}


Table 3: Result Table Seikai

\begin{tabular}{|c|c|c|c|}
\hline Seikai & Method 1 & Method2 & Method 3 \\
\hline \hline 2D MEAN[m] & 1.8994 & 0.3442 & 0.3506 \\
\hline 2D STD[m] & 0.9404 & 0.8622 & 1.0912 \\
\hline 2D RMS[m] & 2.1193 & 0.9281 & 1.1457 \\
\hline UP MEAN[m] & -6.6546 & 0.4533 & 0.5107 \\
\hline UP STD[m] & 1.8220 & 0.8099 & 1.0256 \\
\hline UP RMS[m] & 6.8993 & 1.4951 & 1.5364 \\
\hline
\end{tabular}

Table 4: Result Table Wakkanai

\begin{tabular}{|c|c|c|c|}
\hline Wakkanai & Method 1 & Method2 & Method 3 \\
\hline \hline 2D MEAN[m] & 1.0960 & 0.1690 & 0.3302 \\
\hline 2D STD[m] & 1.0225 & 0.8095 & 0.9059 \\
\hline 2D RMS[m] & 1.4987 & 0.8266 & 0.9639 \\
\hline UP MEAN[m] & -4.1493 & 1.4281 & 1.1806 \\
\hline UP STD[m] & 1.8042 & 0.9863 & 1.0935 \\
\hline UP RMS[m] & 4.5243 & 1.7353 & 1.6090 \\
\hline
\end{tabular}

\subsection{Positioning Results for a Long Time Span}

Next, the results of the positioning errors in horizontal plane and up direction at Kushimoto GEONET station during January and June are shown in Figs. 1114. In Figs. 11-14, $\bigcirc$ symbols with blue lines show the RMS value of the positioning errors with the original Klobuchar Model (Method 1). $\nabla$ with red lines are the results with refined Klobuchar Model (Method 2). * with black lines are the results with previous day's refined Klobuchar Model (Method 3). Fig. 10 shows the refined $D C$ parameters during January $(\bigcirc$ symbols with red line) and during June $(\bigcirc$ symbols with blue line).

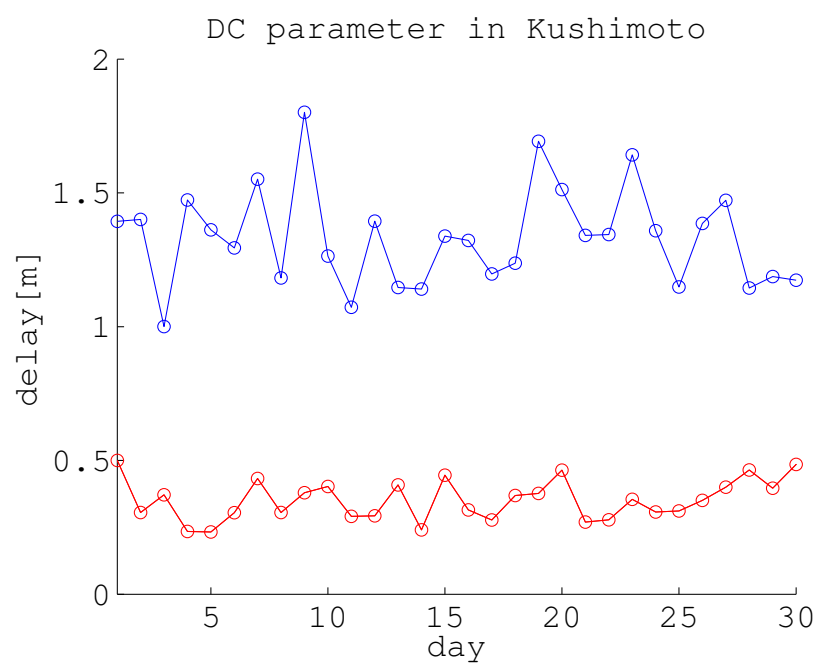

Fig. 10: DC parameter

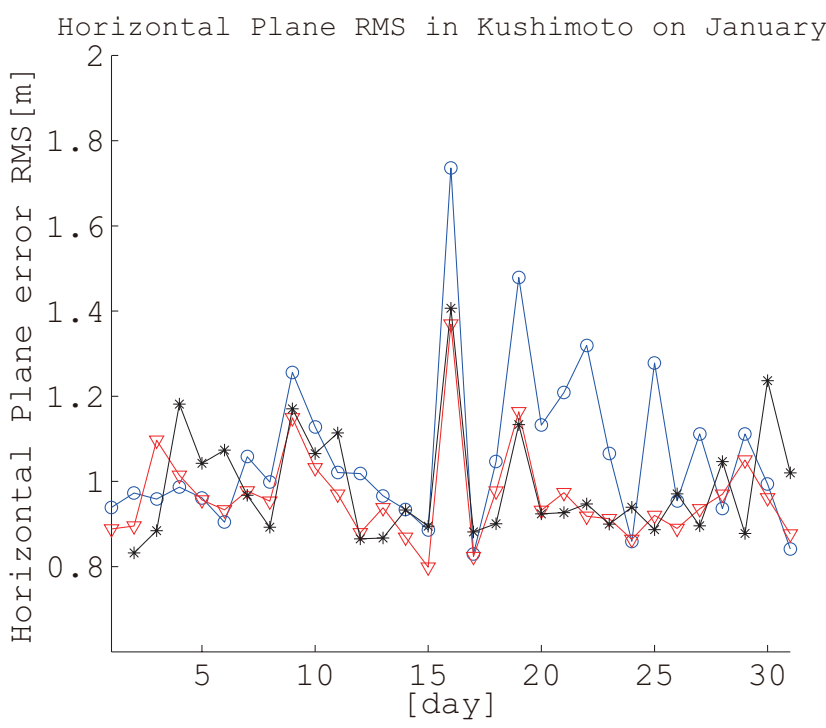

Fig. 11: Error of horizontal plane RMS on January

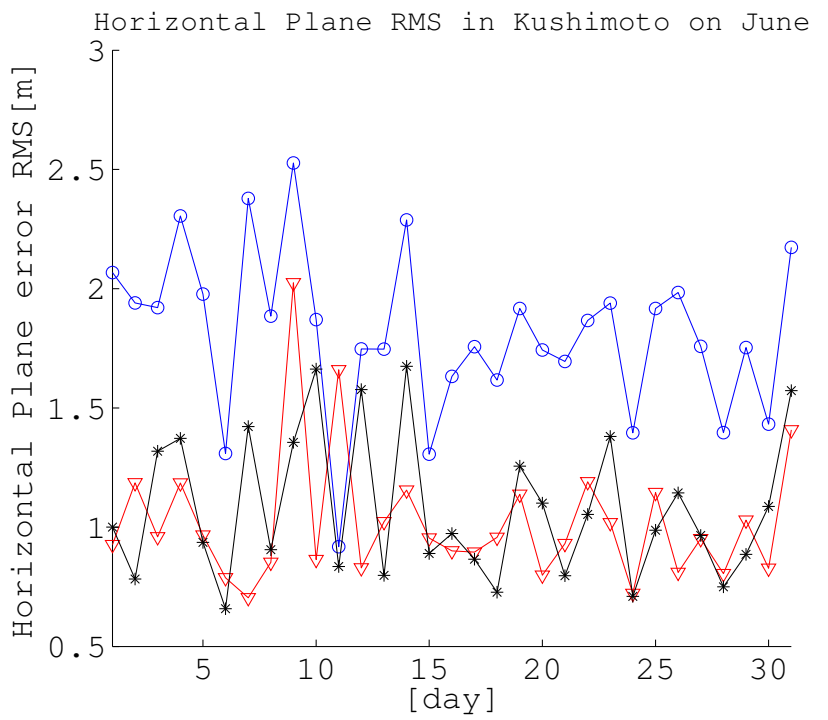

Fig. 12: Error of horizontal plane RMS on June

From Fig.10, it can be seen that the DC parameter in summer season is greater than that in winter season. Although the constant delay of $5.0 \times 10^{-9}$ [sec] is applied in the original Klobuchar model, it is quite better that the DC parameter is estimated seasonally. From Figs.11 and 13, we can see that the error of horizontal plane and up directions were almost improved with refined Klobuchar method on January whereas from Figs.12 and 14, we can see that the error of horizontal plane and up directions were sometimes worse than original coefficients on June. From Figs.11 to 13, it seems that the error of horizontal plane and up direction with previous day's refined coefficients were almost same as with refined coefficients on January whereas on 


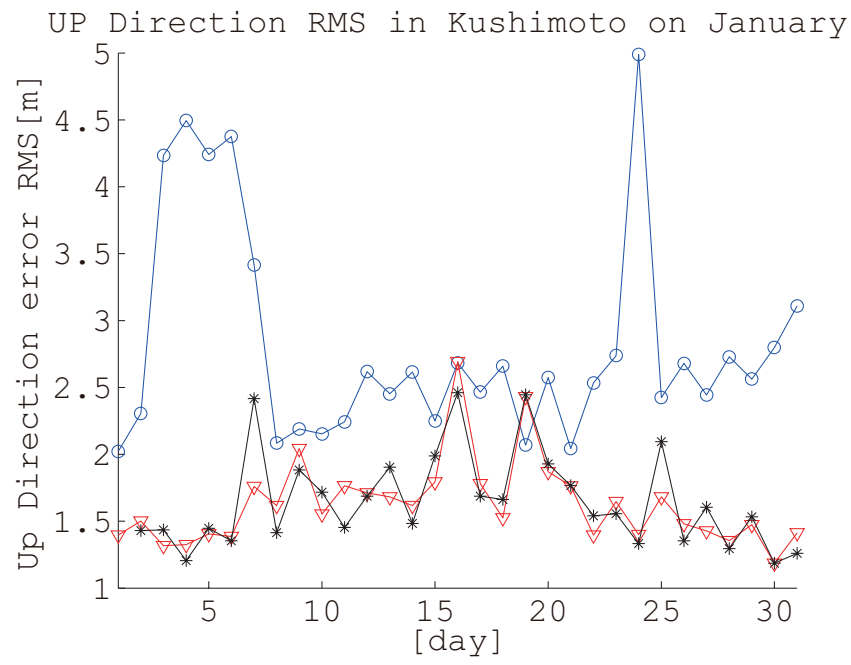

Fig. 13: Error of up direction RMS on January

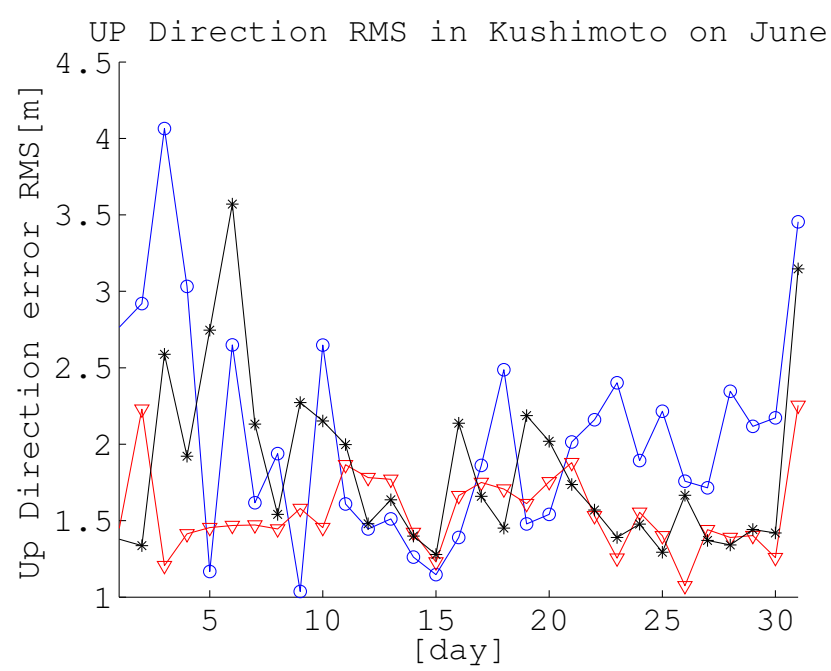

Fig. 14: Error of up direction RMS on June

June the error of horizontal plane and up direction with previous day's refined coefficients were often worse than with original coefficients.

\section{Conclusions}

In this paper, the methods to refine the Klobuchar ionospheric coefficients using SCHA model have been proposed. Experiments result show that refining coefficients, calculated using the proposed method in this paper, can achieve better correction for the effects of the ionospheric delay. This refining method needs SCHA Model coefficients, therefore in actual positioning can use only previous day's refined coefficients. In method 3 result show the error of horizontal plane and up direction with previous day's refined coefficients were almost better than with original model. This result improved the refined coefficients had adequate accuracy for using to actual positioning.

\section{Refferences}

[1] M. Atsushi, H. Kazuaki, I. Minoru, A. Naoki : Estimate the Ionospheric vertical delay in augmentation data of SBAS, ENRI papers, No. 93 (1999).

[2] S. Otsuki, M. Kamimura, M. Ohashi, Y. Kubo and S. Sugimoto: Local Models for Ionospheric VTEC Estimation Based on GR Models and Spherical Cap Harmonic Analysis, Proc. of the International Symposium on GPS/GNSS 2010 (IGNSS 2010), pp. 445-450, Taiwan (2010).

[3] J. A. Klobuchar: Ionospheric Time-Delay Algorithm for Single-Frequency GPS Users, IEEE Transactions on Aerospace and Electronic Systems, Vol. AES-23, No. 3 (1987).

[4] A. Naoki, H. Kazuaki, I. Minory, M. Atsushi: Correction of the Ionospheric Delay on GLONASS using the GPS Navigation Message, ELECTRONIC NAVIGATION RESEARCH INSTITUTE PAPERS, No. 93 August (1999).

[5] S. Sugimoto, Y. Kubo, S. Fujita, T. Imamura and T. Kazuno: Ionospheric Estimation over Japan Based on GNSS Regression Models and GEONRT Data, Proc. ION GNSS 2007, pp. 2346-2356, Sept. (2007).

[6] S. Sugimoto and Y. Kubo: GNSS Regressive Models and Precise Point Positioning, Proc. of 36th ISCIE Int. Symp. on Stochastic Systems Theory and Its Applications, pp. 159-164, Saitama, Japan, Nov. (2004).

[7] S. Fujita, H. Yamamoto, T. Iura, Y. Kubo and S. Sugimoto: Modeling Ionosphere VTEC over Japan Based on GNSS Regression Models and GEONET, Int. of Innovative Computing, Information and Control, pp. 155-169, Vol. 6, No. 1, Jan. (2010).

[8] S. Sugimoto and Y. Kubo : Unified Methods of Point and Relative Positioning Based on GNSS Regression Equations, Proc. of 2007 ION National Technical Meeting, pp. 881-891, San Diego, CA, Jan. (2007)

[9] Y. Yuan, X, Huo, J. Ou, K. Zhang, Y. Chai, D. Wen, R. Grenfell: Refining the Klobuchar Ionospheric Coefficients Based on GPS Observations, IEEE Transactions on Aerospace and Electronic Systems, Vol. AES-44, No. 4 (2008).

[10] T. Sakai: Bias Error Calibration for Observing Ionosphere by GPS Network, Trans. of IEICE $B$, Vol. J88-B, No. 12, pp. 2382-2389 (2005), in Japanese. 